that received faecal microbiota from healthy individuals. Wild-type animals that received faecal microbiota from people with Parkinson's disease did not show movement abnormalities. Together, these results provide compelling evidence that gut microbes have a role in disease pathology.

Sampson and colleagues' findings raise several questions. How could short-chain fatty acids exert their effects on Parkinson's disease, given that the FFAR2 receptor protein for these molecules has not been observed to be expressed in the brain ${ }^{11}$ ? Does this mean that other cells are involved that express FFAR2 and produce metabolites that modify disease outcome, or can short-chain fatty acids influence brain cells without requiring the presence of FFAR2 receptors? Another question is whether the host microbiota shapes any other forms of neurodegeneration, as might be inferred from the previous finding that the amount of disease-associated amyloid protein is altered after antibiotic treatment in a mouse model of Alzheimer's disease ${ }^{16}$.

Other aspects of these results are worth considering. Absence of the host microbiota changes the permeability of the blood-brain barrier in mice and affects brain cells other than microglia, ${ }^{9,10}$, which might account for the reduced Parkinson's symptoms observed by Sampson and colleagues. The synthesis of several neurotransmitters, including dopamine, is also affected by the absence of the host microbiota ${ }^{9}$. However, the authors did not investigate whether their model mice had altered numbers of dopamine-expressing neurons or dopamine levels.

Sampson et al. find that the gut microbiota is intimately involved in the pathogenesis of Parkinson's disease, and they suggest that this involvement is mediated by regulation of the activation status of microglia. Knowing how compounds generated by bacteria can shape the immune response in the brain and the development as well as the outcome of neurodegenerative disease will undoubtedly contribute to our understanding of Parkinson's disease, and perhaps open other therapeutic avenues for treating this devastating condition.

\section{Daniel Erny and Marco Prinz are in}

the Faculty of Medicine, Institute of

Neuropathology, University of Freiburg,

D-79106 Freiburg, Germany. D.E. is also in

the Berta-Ottenstein programme, University

of Freiburg. M.P. is also in the BIOSS Centre

for Biological Signalling Studies, University of

Freiburg.

e-mail:marco.prinz@uniklinik-freiburg.de

1. Sender, R., Fuchs, S. \& Milo, R. Cell 164, 337-340 (2016).

2. Hooper, L. V. \& Gordon, J. I. Science 292, 1115-1118 (2001).

3. Sampson, T. R. et al. Cell 167, 1469-1480 (2016).

4. de Lau, L. M. L. \& Breteler, M. M. B. Lancet Neurol. 5, 525-535 (2006).
5. Braak, H., Rüb, U., Gai, W. P. \& Del Tredici, K. J. Neural Transm. 110, 517-536 (2003).

6. Prinz, M. \& Priller, J. Nature Rev. Neurosci. 15, 300-312 (2014).

7. Goldmann, T. et al. Nature Neurosci. 16, 1618-1626 (2013).

8. Gao, L. et al. J. Exp. Med. 212, 469-480 (2015).

9. Diaz Heijtz, R. et al. Proc. Natl Acad. Sci. USA 108 , 3047-3052 (2011)

10.Braniste, V. et al. Sci. Transl. Med. 6, 263 ra158 (2014).

11.Erny, D. et al. Nature Neurosci. 18, 965-977 (2015).

12.Svensson, E. et al. Ann. Neurol. 78, 522-529 (2015)

13.Chesselet, M.-F. et al. Neurotherapeutics 9, 297-314 (2012).

14.Smith, P. M. et al. Science 341, 569-573 (2013).

15.Scheperjans, F. et al. Mov. Disord. 30, 350-358 (2015).

16.Minter, M. R. et al. Sci. Rep. 6, 30028 (2016).

This article was published online on 12 April 2017.

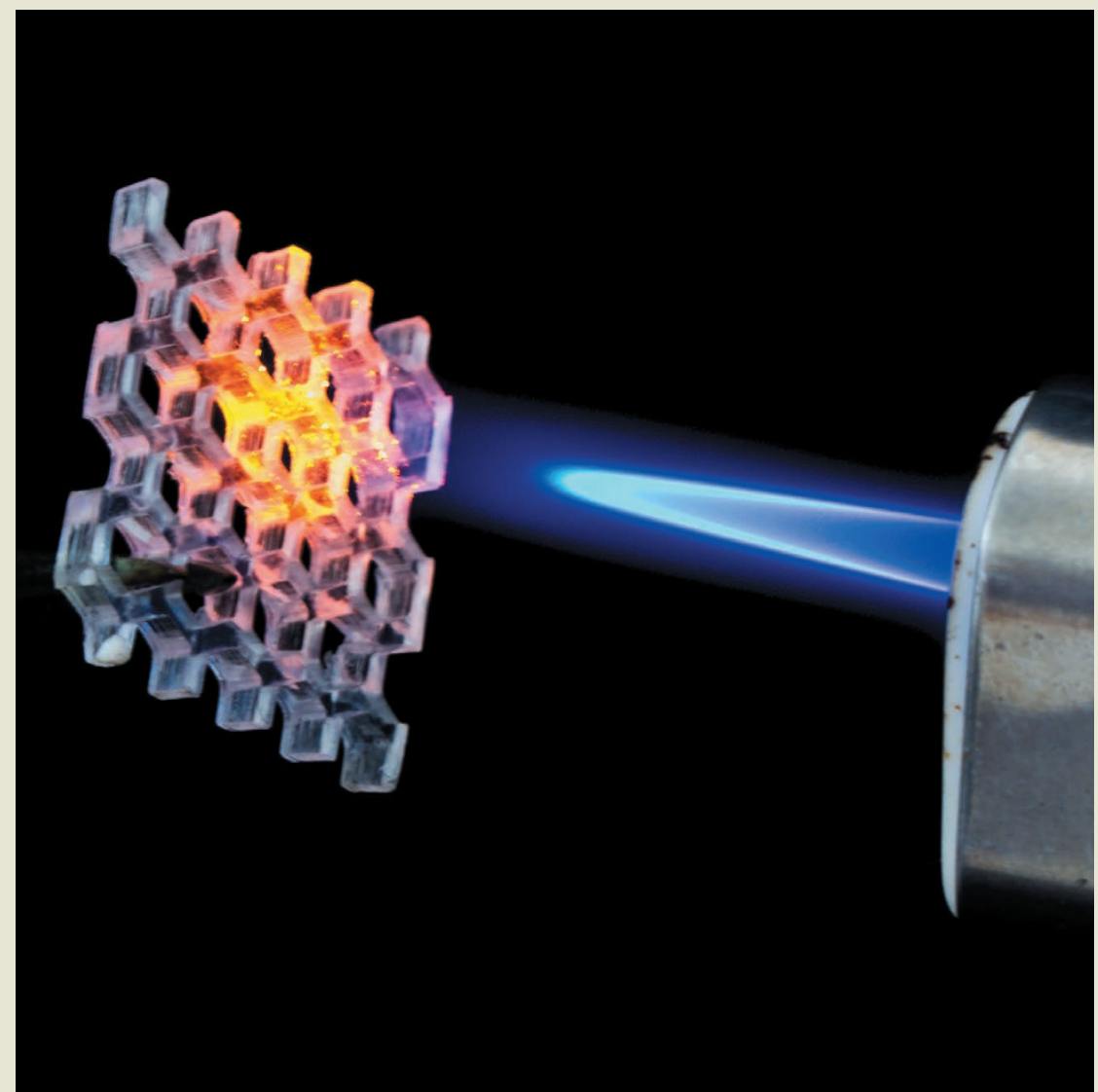

MATERIALS SCIENCE

\title{
How to print glass
}

From time immemorial, optically transparent fused silica glasses have been treasured for their physical, chemical and aesthetic qualities. When it comes to producing and patterning such glasses, the image that typically springs to mind is of a high-temperature furnace and a sweaty glass-blower skilfully moulding the molten starting material. But are such extreme working conditions and manual dexterity strictly necessary? Perhaps not. In this issue, Kotz et al. describe a method for fabricating high-quality glass components of arbitrary complexity using a standard 'stereolithographic' 3D printer (F. Kotz et al. Nature 544, 337-339; 2017).
Key to the authors' approach is the development of a fluid composite consisting of silica nanoparticles dispersed in a monomeric matrix that is polymerized during the printing process. Kotz et al. carefully chose the ingredients of this nanocomposite such that when the printed, polymerized structure is sintered - fused at temperatures of about $1,300^{\circ} \mathrm{C}$ - it yields a glass of high optical quality. The glass faithfully replicates the printed edifice and has impressive thermal resistance (pictured). Its intricate features are limited only by the resolution of the printer (a few tens of micrometres in this case). Karl Ziemelis 